\title{
Fast state estimation in linear time-invariant systems: an algebraic approach
}

\author{
Yang TIAN, Thierry FLOQUET and Wilfrid PERRUQUETTI
}

\begin{abstract}
In this note, an algebraic approach for state estimation of linear time invariant systems is developed. This approach is based on the following mathematical tools: Laplace transform, Leibniz formula and operational calculus. A generalized expression of the state variables in function of the integrals of the output and the input is obtained. The example of a DC motor system and simulation results are given to illustrate the performance of the proposed approach.
\end{abstract}

\section{INTRODUCTION}

State estimation of linear systems has been extensively studied in the literature because the associated problems are of great interest for engineers. Indeed, the state is not always available by direct measurement and a state observer (a dynamic auxiliary system), which gives a complete estimate based on measurements and inputs, must be designed.

An observer can be constructed if the system is observable, i.e. if any initial state $x\left(t_{0}\right)$ at $t_{0}$ can be determined from the knowledge of the system output $y$ and the control $u$. In the context of deterministic linear finite-dimensional timeinvariant systems, an observer was first introduced by Luenberger [8] leading to the asymptotic estimation of the state. In the case of linear system with noise, where stochastic phenomena appear, a so-called Kalman filter can be designed [6], [7].

The purpose of this article is to design a fast reconstructor of the state for linear time-invariant systems using an algebraic approach, which is an extension of recent works from Fliess and Sira-Ramirez [4], [5]. As a result, the process of estimation is given by an exact formula, rather than by an auxiliary dynamic system. In this approach, the successive time derivatives of the output are expressed in function of the integral of the output $y$ itself and of the input $u$ so that the state can be estimated in function of the integral of $y$, the input $u$ and a finite number of its time derivatives. The proposed method exhibits the following features: (i) independence of tunable parameters, observer gains, (ii) independence of noise models and its parameterization, (iii) formal and very fast computer computations, (iv) robustness properties with respect to different noises. The third order example of a DC motor highlights the efficacy of the proposed approach.

The authors are with LAGIS (CNRS, UMR 8146), École Centrale de Lille, BP 48, Cité Scientifique, 59650 Villeneuve d'Ascq, France and with Équipe-Projet ALIEN, INRIA Lille - Nord Europe. Yang TIAN is also with Institute of Electric Engineering, HoHai University, China. yang.tian@inria.fr, thierry.floquetdec-lille.fr,

wilfrid.perruquettidec-lille.fr

\section{PROBLEM STATEMENT}

Consider the linear time invariant system:

$$
\left\{\begin{aligned}
\dot{x} & =A x+B u \\
y & =C x
\end{aligned}\right.
$$

where $x \in \mathbb{R}^{n}$ is the state, $u \in \mathbb{R}^{m}$ is the input and $y \in \mathbb{R}^{d}$ is the output. $A \in \mathbb{R}^{n \times n}, B \in \mathbb{R}^{n \times m}$ and $C \in \mathbb{R}^{d \times n}$ are constant matrices. The system (1) is assumed to be observable, i.e. it satisfies the Kalman rank condition ([6]):

$$
\operatorname{Rank} \mathbf{O}_{(A, C)}=n
$$

where $\mathbf{O}_{(A, C)}$ is the so-called matrix of observability:

$$
\mathbf{O}_{(A, C)}=\left[C^{T},(C A)^{T}, \ldots,\left(C A^{n-1}\right)^{T}\right]^{T} .
$$

In this article, an algebraic viewpoint for the state estimation problem is taken. In [1], [3], observability is discussed from a differential algebra standpoint. In this context, the observability of the system (1) is equivalent to the possibility to express all the variables of the system (in particular all the state variables) as combinations of the components of the input, the output and of their time derivatives up to a finite order. This criterion is valid not only for linear systems, but also for nonlinear systems [2].

Here, observable monovariable systems are considered, that is to say: $u \in \mathbb{R}$ and $y \in \mathbb{R}$. It is aimed to estimate the state $x$ in a fast way and on the basis of possibly noisy measurements. For this, exact expression of the state are derived in function of the integral of the output. The influence of measurement noises can also be reduced with the integral operation which has a filtering effect.

For the sake of convenience, useful formulas which will be used hereafter are introduced:

$$
\begin{aligned}
& \text { (i) } \mathscr{L}^{-1}\left(\frac{1}{s^{l}} \frac{d^{k} Y(s)}{d s^{k}}\right) \\
& =\left\{\begin{array}{cc}
\int \ldots \int\left(-\tau_{1}\right)^{k} y\left(\tau_{1}\right) d \tau_{1} \ldots d \tau_{l}, & \text { if } l \geq 1 \\
\frac{d^{l}\left((-t)^{k} y(t)\right)}{d t^{l}}, & \text { if } l \leq 0
\end{array}\right. \\
& \text { (ii) } \int \ldots \int y\left(\tau_{1}\right) d \tau_{1} \ldots d \tau_{l}=\int_{0}^{t} \frac{(t-\tau)^{l-1} y(\tau)}{(l-1) !} d \tau \\
& \text { (iii) }(f * g)(t)=\int_{0}^{t} f(t-\lambda) g(\lambda) d \lambda \\
& \text { (iv) } \int_{0}^{t} \delta\left(\lambda-\lambda_{0}\right) f(\lambda) d \lambda=f\left(\lambda_{0}\right)
\end{aligned}
$$

where $\delta(t)$ is a Dirac distribution. 


\section{ALGEBRAIC APPROACH OF STATE ESTIMATION}

¿From system (1), one can obtain the input-output relationship:

$$
\sum_{i=0}^{n} a_{i} y^{(i)}=\sum_{i=0}^{m} b_{i} u^{(i)}
$$

where $a_{n}=1$ and $m<n$, and one can express the state $x$ in function of $y, u$ and their time derivatives:

$$
x(t)=\mathbf{O}_{(A, C)}^{-1}\left[\left(\begin{array}{c}
y \\
\dot{y} \\
y^{(2)} \\
\vdots \\
y^{(n-1)}
\end{array}\right)-\mathbf{M}\left(\begin{array}{c}
u \\
\dot{u} \\
u^{(2)} \\
\vdots \\
u^{(n-2)}
\end{array}\right)\right]
$$

where

$$
\mathbf{M}=\left(\begin{array}{cccc}
0 & 0 & \ldots & 0 \\
C B & 0 & \ldots & 0 \\
C A B & C B & \ldots & 0 \\
\vdots & \vdots & \vdots & \vdots \\
C A^{n-2} B & C A^{n-3} B & \ldots & C B
\end{array}\right)
$$

The matrix of observability is invertible since the system is observable. Thus, one can recover the state of the system if one has the knowledge of the outputs and a finite number of their time derivatives. In the following, an algebraic method is developed to obtain a fast and accurate estimate of those variables.

Theorem 1: For the linear time invariant monovariable system (1), the estimates of the successive time derivatives of the measured output $y$ are given by:

$$
\begin{gathered}
\left(\begin{array}{c}
y_{e}^{(1)}(t) \\
y_{e}^{(2)}(t) \\
y_{e}^{(3)}(t) \\
\vdots \\
y_{e}^{(n-1)}(t)
\end{array}\right)=-\frac{1}{(-t)^{n}} \tilde{R}\left(\begin{array}{c}
y_{e}(t) \\
y_{e}^{(1)}(t) \\
y_{e}^{(2)}(t) \\
\vdots \\
y_{e}^{(n-2)}(t)
\end{array}\right) \\
+\frac{1}{(-t)^{n}}\left(\sum_{i=0}^{m} b_{i}\left(\begin{array}{c}
D_{i, 1}(u) \\
D_{i, 2}(u) \\
D_{i, 3}(u) \\
\vdots \\
D_{i, n-1}(u)
\end{array}\right)-\sum_{i=0}^{n} a_{i}\left(\begin{array}{c}
\tilde{A}_{i, 1}(y) \\
\tilde{A}_{i, 2}(y) \\
\tilde{A}_{i, 3}(y) \\
\vdots \\
\tilde{A}_{i, n-1}(y)
\end{array}\right)\right)
\end{gathered}
$$

with

$$
\begin{aligned}
& y_{e}(t)=\frac{\sum_{i=0}^{m} b_{i}}{(-t)^{n}}\left(\sum_{\substack{j=0 \\
n \leq i+j}}^{n} \frac{c_{i, j} \int_{0}^{t}(t-\tau)^{2 n-i-j-1}(-\tau)^{j} u(\tau) d \tau}{(2 n-i-j-1) !}\right) \\
& -\frac{\sum_{i=0}^{n} a_{i}}{(-t)^{n}}\left(\sum_{\substack{j=0 \\
n \leq i+j<2 n}}^{n} \frac{c_{i, j} \int_{0}^{t}(t-\tau)^{2 n-i-j-1}(-\tau)^{j} y(\tau) d \tau}{(2 n-i-j-1) !}\right)
\end{aligned}
$$

and

$$
\begin{gathered}
\tilde{R}=\left(\begin{array}{ccccc}
\alpha_{1,1} & 0 & 0 & \ldots & 0 \\
\alpha_{2,1} & \alpha_{2,2} & 0 & \ldots & 0 \\
\alpha_{3,1} & \alpha_{3,2} & \alpha_{3,3} & \ldots & 0 \\
\vdots & \vdots & \vdots & \vdots & \vdots \\
\alpha_{n-1,1} & \alpha_{n-1,2} & \alpha_{n-1,3} & \ldots & \alpha_{n-1, n-1}
\end{array}\right) \\
\alpha_{p, l}=\sum_{i=n-p+l-1}^{n} a_{i=2 n-p+l-1-i} \sum_{i, j}^{n} \tilde{r}_{l-1, w}+\tilde{d}_{p, l-1} \\
c_{i, j}=\left(\begin{array}{c}
n \\
j
\end{array}\right) \frac{i !}{(i+j-n) !}, \quad w=p+i+j-2 n \\
\tilde{r}_{g, w}=\left(\begin{array}{c}
w \\
g
\end{array}\right) \frac{j !(-1)^{j} t^{j-w+g}}{(j-w+g) !}, \quad \tilde{d}_{p, k}=\left(\begin{array}{l}
p \\
k
\end{array}\right) \frac{n !(-1)^{n} t^{n-p+k}}{(n-p+k) !} \\
\tilde{A}_{i, p}(y)=\frac{\sum_{j=0}^{n} c_{i, j} \frac{\int_{0}^{t}(t-\tau)^{-w-1}(-\tau)^{j} y(\tau) d \tau}{(-w-1) !}}{p-n \leq w<0} \\
D_{i, p}(u)=\frac{(-1)^{i}}{(n-p-1) !} \int_{0}^{t}\left\{(t-\lambda)^{n-p-1}(-\lambda)^{n}\right\}^{(i)} u(\lambda) d \lambda
\end{gathered}
$$

\section{Proof}

The method is divided into three steps.

Step 1: Express $y_{e}$, the estimate of $y$, in function of the integral of the output $y$ and the input $u$.

a) Apply the Laplace transform to the I/O relationship (2).

$$
\begin{aligned}
\sum_{i=0}^{n} a_{i}\left(s^{i} y_{(s)}-s^{i-1} y_{(0)}-\ldots-y_{(0)}^{(i-1)}\right) & \\
= & \sum_{i=0}^{m} b_{i}\left(s^{i} u_{(s)}-s^{i-1} u_{(0)}-\ldots-u_{(0)}^{(i-1)}\right)
\end{aligned}
$$

b) Algebraic manipulations.

Deriving the preceding expression $n$ times with respect to $s$ to eliminate the initial conditions and using the Leibniz formula:

$$
\frac{d^{h}(x(s) y(s))}{d s^{h}}=\sum_{j=0}^{h}\left(\begin{array}{l}
h \\
j
\end{array}\right) \frac{d^{h-j}(x(s))}{d s^{h-j}} \frac{d^{j}(y(s))}{d s^{j}},
$$

and the relation:

$$
\frac{d^{k}\left(s^{l}\right)}{d s^{k}}=\left\{\begin{array}{cc}
\frac{l !}{(l-k) !} s^{l-k}, & \text { if } 0<k \leq l \\
0, & \text { if } 0<l<k \\
\frac{(-1)^{k}(k-l-1) !}{(-l-1) !} s^{l-k}, & \text { if } l<0<k
\end{array}\right.
$$

one gets:

$$
\begin{aligned}
& \sum_{i=0}^{n} a_{i}\left(\sum_{\substack{j=0 \\
i+j \geq n}}^{n}\left(\begin{array}{l}
n \\
j
\end{array}\right) \frac{i ! s^{i+j-n}}{(i+j-n) !} \frac{d^{j}(y(s))}{d s^{j}}\right) \\
& =\sum_{i=0}^{m} b_{i}\left(\sum_{\substack{j=0 \\
i+j \geq n}}^{n}\left(\begin{array}{l}
n \\
j
\end{array}\right) \frac{i ! s^{i+j-n}}{(i+j-n) !} \frac{d^{j}(u(s))}{d s^{j}}\right)
\end{aligned}
$$


Set $c_{i, j}=\left(\begin{array}{l}n \\ j\end{array}\right) \frac{i !}{(i+j-n) !}$ and multiply each side of (7) by $s^{-n}$ :

$$
\sum_{i=0}^{n} a_{i} \sum_{\substack{j=0 \\ i+j \geq n}}^{n} \frac{c_{i, j}}{s^{2 n-i-j}} \frac{d^{j}(y(s))}{d s^{j}}=\sum_{i=0}^{m} b_{i} \sum_{\substack{j=0 \\ i+j \geq n}}^{n} \frac{c_{i, j}}{s^{2 n-i-j}} \frac{d^{j}(u(s))}{d s^{j}}
$$

c) Return to time domain.

Using the two formulas $(i)$ and (ii), one gets:

$$
\mathscr{L}^{-1}\left(\frac{1}{s^{l}} \frac{d^{k} Y(s)}{d s^{k}}\right)=\int_{0}^{t} \frac{(t-\tau)^{l-1}(-\tau)^{k} y(\tau)}{(l-1) !} d \tau, \quad l \geq 1
$$

Thus,

$$
\begin{aligned}
& \mathscr{L}^{-1}\left(\frac{1}{s^{2 n-i-j}} \frac{d^{j}(y(s))}{d s^{j}}\right)= \\
&\left\{\begin{array}{cc}
\int_{0}^{t} \frac{(t-\tau)^{2 n-i-j-1}(-\tau)^{j} y(\tau)}{(2 n-i-j) !} d \tau, & \text { if } 2 n-i-j \geq 1 \\
(-t)^{n} y(t), & \text { if } j=i=n
\end{array}\right.
\end{aligned}
$$

and the inverse Laplace transform of (8) is given by:

$$
\begin{aligned}
& \sum_{i=0}^{n} a_{i}\left(\sum_{\substack{j=0 \\
n \leq i+j<2 n}}^{n} \frac{c_{i, j} \int_{0}^{t}(t-\tau)^{2 n-i-j-1}(-\tau)^{j} y(\tau) d \tau}{(2 n-i-j-1) !}\right) \\
& +(-t)^{n} y(t) \\
& =\sum_{i=0}^{m} b_{i}\left(\sum_{\substack{j=0 \\
n \leq i+j}}^{n} \frac{c_{i, j} \int_{0}^{t}(t-\tau)^{2 n-i-j-1}(-\tau)^{j} u(\tau) d \tau}{(2 n-i-j-1) !}\right)
\end{aligned}
$$

Then, the estimate $y_{e}$ can be expressed as in (5) and only depends on the integral of $y$ and $u$.

Step 2: Express $y_{e}^{(p)}$ in function of the integral of $y$ and $u$ (the integral action attenuates the measurement noises).

a) Algebraic manipulations.

Apply the Laplace transform to the I/O relationship (2), derive the obtained expression $n$ times with respect to $s$ and multiply each side by $s^{-(n-p)}$ :

$$
\sum_{i=0}^{n} a_{i} \underbrace{\frac{1}{s^{n-p}} \frac{d^{n} \mathscr{L}\left(y^{(i)}(t)\right)}{d s^{n}}}_{\widetilde{C}_{i, p}}=\sum_{i=0}^{m} b_{i} \underbrace{\frac{1}{s^{n-p}} \frac{d^{n} \mathscr{L}\left(u^{(i)}(t)\right)}{d s^{n}}}_{\widetilde{D}_{i, p}}
$$

With similar manipulations as in the first step, one has:

$$
\widetilde{C}_{i, p}=\sum_{\substack{j=0 \\ i+j \geq n}}^{n} c_{i, j} s^{w} \frac{d^{j}(y(s))}{d s^{j}}, \quad w=p+i+j-2 n
$$

b) Return to time domain.

By using the formulas (i) and (ii), one gets:

$\mathscr{L}^{-1}\left(s^{w} \frac{d^{j}(y(s))}{d s^{j}}\right)=\left\{\begin{array}{cl}\int_{0}^{t} \frac{(t-\tau)^{-w-1}(-\tau)^{j} y(\tau)}{(-w-1) !} d \tau, & \text { if } w<0 \\ \frac{\left.d^{w}((-t))^{j} y(t)\right)}{d t^{w}}, & \text { if } w \geq 0\end{array}\right.$
Thus:

$$
\mathscr{L}^{-1}\left(\tilde{C}_{i, p}\right)=\tilde{A}_{i, p}(y)+\sum_{\substack{j=0 \\ 0 \leq w<p}}^{n} c_{i, j} \frac{d^{w}\left((-t)^{j} y(t)\right)}{d t^{w}}+\frac{d^{p}\left((-t)^{n} y(t)\right)}{d t^{p}}
$$

$\tilde{A}_{i, p}(y)=\sum_{\substack{j=0 \\ p-n \leq w<0}}^{n} c_{i, j} \frac{\int_{0}^{t}(t-\tau)^{-w-1}(-\tau)^{j} y(\tau) d \tau}{(-w-1) !}$

Applying the Leibniz formula and the relation (6), one gets:

$$
\begin{aligned}
& \frac{d^{w}\left((-t)^{j} y(t)\right)}{d t^{w}}=\sum_{g=0}^{w}\left(\begin{array}{c}
w \\
g
\end{array}\right) \frac{j !(-1)^{j} t^{j-w+g}}{(j-w+g) !} \frac{d^{g}(y(t))}{d t^{g}} \\
& \frac{d^{p}\left((-t)^{n} y(t)\right)}{d t^{p}}=(-t)^{n} y^{(p)}(t)+\sum_{k=0}^{p-1}\left(\begin{array}{l}
p \\
k
\end{array}\right) \frac{n !(-1)^{n} t^{n-p+k}}{(n-p+k) !} \frac{d^{k}(y(t))}{d t^{k}}
\end{aligned}
$$

Using the results of (10), (11) and (12), one gets:

$$
\sum_{i=0}^{n} a_{i} \mathscr{L}^{-1}\left(\tilde{C}_{i, p}\right)=\sum_{i=0}^{n} a_{i} \tilde{A}_{i, p}(y)+\Gamma_{p}(y)+(-t)^{n} y^{(p)}(t)
$$

with

$$
\begin{gathered}
\Gamma_{p}(y)=\sum_{i=0}^{n} a_{i} \sum_{\substack{j=0 \\
0 \leq w<p}}^{n} c_{i, j} \sum_{g=0}^{w}\left(\begin{array}{l}
w \\
g
\end{array}\right) \frac{j !(-1)^{j} t^{j-w+g}}{(j-w+g) !} \frac{d^{g}(y(t))}{d t^{g}} \\
+\sum_{k=0}^{p-1}\left(\begin{array}{l}
p \\
k
\end{array}\right) \frac{n !(-1)^{n} t^{n-p+k}}{(n-p+k) !} \frac{d^{k}(y(t))}{d t^{k}}
\end{gathered}
$$

It can be shown that

$$
\begin{aligned}
\Gamma_{p}(y)= & {\left[a_{n}\left(c_{n, n-p} \tilde{r}_{0,0}+c_{n, n-p+1} \tilde{r}_{0,1}+\ldots+c_{n, n-1} \tilde{r}_{0, p-1}\right)\right.} \\
& +a_{n-1}\left(c_{n-1, n-p+1} \tilde{r}_{0,0}+\ldots+c_{n-1, n} \tilde{r}_{0, p-1}\right)+\ldots \\
& \left.+a_{n-p} c_{n-p, n} \tilde{r}_{0,0}\right] y+\tilde{d}_{p, 0} y \\
+ & {\left[a_{n}\left(c_{n, n-p+1} \tilde{r}_{1,1}+c_{n, n-p+2} \tilde{r}_{1,2}+\ldots+c_{n, n-1} \tilde{r}_{1, p-1}\right)\right.} \\
& +a_{n-1}\left(c_{n-1, n-p+2} \tilde{r}_{1,1}+\ldots+c_{n-1, n} \tilde{r}_{1, p-1}\right)+\ldots \\
& \left.+a_{n-p+1} c_{n-p+1, n} \tilde{r}_{1,1}\right] y^{(1)}+\tilde{d}_{p, 1} y^{(1)}+\ldots \\
+ & {\left[a_{n} c_{n, n-1} \tilde{r}_{p-1, p-1}+a_{n-1} c_{n-1, n} \tilde{r}_{p-1, p-1}\right] y^{(p-1)} } \\
& +\tilde{d}_{p, p-1} y^{(p-1)} \\
= & \alpha_{p, 1} y+\alpha_{p, 2} y^{(1)}+\ldots+\alpha_{p, p} y^{(p-1)} \\
= & \sum_{l=1}^{p} \alpha_{p, l} y^{(l-1)}
\end{aligned}
$$

with

$$
\alpha_{p, l}=\sum_{i=n-p+l-1}^{n} a_{i} \sum_{j=2 n-p+l-1-i}^{n} c_{i, j} \tilde{r}_{l-1, w}+\tilde{d}_{p, l-1}
$$

In order to express $\mathscr{L}^{-1}\left(\widetilde{D}_{i, p}\right)$, one applies the convolution theorem given by:

$$
\mathscr{L}^{-1}\left(g_{1}(s) g_{2}(s)\right)=g_{1}(t) * g_{2}(t)
$$

This leads to

$$
D_{i, p}(u)=\mathscr{L}^{-1}\left(\widetilde{D}_{i, p}\right)=\frac{t^{n-p-1} \varepsilon(t)}{(n-p-1) !} *(-t)^{n} u^{(i)}(t),
$$


where $\varepsilon(t)$ is the step function.

If $g_{1}$ is a $C^{1}$-function such that $g_{1}(0)=0$ and $g_{2}$ is a $C^{0}$-function, then:

$$
\begin{aligned}
& \int_{0}^{t} g_{1}(t-\lambda) g_{2}(\lambda) d \lambda \\
& =\left[g_{1}(t-\lambda) \int_{0}^{\lambda} g_{2}(\mu) d \mu\right]_{0}^{t}-\int_{0}^{t} \frac{d g_{1}(t-\lambda)}{d \lambda}\left(\int_{0}^{\lambda} g_{2}(\mu) d \mu\right) d \lambda \\
& =\int_{0}^{t} \frac{d g_{1}(t-\lambda)}{d(t-\lambda)}\left(\int_{0}^{\lambda} g_{2}(\mu) d \mu\right) d \lambda .
\end{aligned}
$$

This result can be extended for two distributions $\left(g_{1}, g_{2}\right)$ with left hand side limited supports which implies the existence of the convolution product $g_{1} * g_{2}$ and the following more general result

$$
\int_{0}^{t} g_{1}^{\prime}(t-\lambda) g_{2}(\lambda) d \lambda=\int_{0}^{t} g_{1}(t-\lambda) g_{2}^{\prime}(\lambda) d \lambda
$$

which reads as

$$
g_{1}^{\prime}(t) * g_{2}(t)=g_{1}(t) * g_{2}^{\prime}(t),
$$

where the prime notation denotes the distribution derivation.

Using the formulas (i)-(iv) and (14), one has:

$$
\begin{aligned}
& t^{n-p-1} \varepsilon(t) *(-t)^{n} u^{(i)}(t) \\
& \stackrel{(14)}{=}\left[(n-p-1) t^{n-p-2} \varepsilon(t)+t^{n-p-1} \delta(t)\right] * \int_{0}^{t}\left(-\tau_{1}\right)^{n} u^{(i)} d \tau_{1} \\
& \stackrel{(i i i)(i v)}{=}(n-p-1) t^{n-p-2} \varepsilon(t) * \int_{0}^{t}\left(-\tau_{1}\right)^{n} u^{(i)} d \tau_{1} \\
& \stackrel{(14)}{=}(n-p-1) ! \varepsilon(t) * \int_{0}^{(n-p-1)}(-t)^{n} u^{(i)} \\
& \stackrel{(i i i)}{=}(n-p-1) ! \int_{0}^{(n-p)} \varepsilon\left(t-\tau_{1}\right)\left(-\tau_{1}\right)^{n} u^{(i)} \\
& =(n-p-1) ! \int_{0}^{(n-p)}(-t)^{n} u^{(i)} \\
& \stackrel{(i i)}{=} \int_{0}^{t}\left(t-\tau_{1}\right)^{n-p-1}\left(-\tau_{1}\right)^{n} u^{(i)} d \tau_{1}
\end{aligned}
$$

where the following notations were used:

$$
\begin{aligned}
& \int_{0}^{(k)}(-t)^{n} \phi=\int_{0}^{t} \ldots \int_{0}^{\tau_{2}}\left(-\tau_{1}\right)^{n} \phi\left(\tau_{1}\right) d \tau_{1} \ldots d \tau_{k} ; \\
& \int_{0}^{(k)} \varepsilon\left(t-\tau_{1}\right)\left(-\tau_{1}\right)^{n} \phi \\
& \quad=\int_{0}^{t} \ldots \int_{0}^{\tau_{2}} \varepsilon\left(\tau_{2}-\tau_{1}\right)\left(-\tau_{1}\right)^{n} \phi\left(\tau_{1}\right) d \tau_{1} \ldots d \tau_{k} ;
\end{aligned}
$$

So

$$
D_{i, p}(u)=\int_{0}^{t} \frac{(t-\lambda)^{n-p-1}}{(n-p-1) !}(-\lambda)^{n} u^{(i)}(\lambda) d \lambda .
$$

Then, applying the integration by parts generalized for the function of class $C^{i}$ :

$$
\begin{aligned}
& \int_{a}^{b} f(\lambda) g^{(i)}(\lambda) d \lambda \\
= & {\left[\sum_{k=0}^{i-1}(-1)^{k} f^{(k)}(\lambda) g^{(i-1-k)}(\lambda)\right]_{a}^{b}+(-1)^{i} \int_{a}^{b} f^{(i)}(\lambda) g(\lambda) d \lambda }
\end{aligned}
$$

one gets:

$D_{i, p}(u)=\frac{1}{(n-p-1) !}\left(\left[\sum_{j=0}^{i-1}(-1)^{j} \widetilde{F}_{p, j} u^{(i-j-1)}(\lambda)\right]_{0}^{t}+(-1)^{i} \int_{0}^{t} \widetilde{F}_{p, i} u(\lambda) d \lambda\right)$ where

$$
\begin{aligned}
\widetilde{F}_{p, j} & =\left\{(t-\lambda)^{n-p-1}(-\lambda)^{n}\right\}^{(j)} \\
& =\left\{\left(\lambda^{2}-t \lambda\right)^{n-p-1}(-\lambda)^{p+1}\right\}^{(j)} \\
& =\sum_{f=0}^{j}\left(\begin{array}{l}
j \\
f
\end{array}\right) \frac{d^{j-f}\left\{\left(\lambda^{2}-t \lambda\right)^{n-p-1}\right\}}{d \lambda^{j-f}} \frac{d^{f}\left\{(-\lambda)^{p+1}\right\}}{d \lambda f} \\
& =\sum_{f=0}^{j}\left(\begin{array}{l}
j \\
f
\end{array}\right) \frac{d^{j-f}\left(\left(\lambda^{2}-t \lambda\right)^{n-p-1}\right)}{d\left(\lambda^{2}-t \lambda\right)^{j-f}} \frac{d\left(\lambda^{2}-t \lambda\right)^{j-f}}{d \lambda \lambda^{j-f}} \frac{d^{f}\left\{(-\lambda)^{p+1}\right\}}{d \lambda f} .
\end{aligned}
$$

Using the relation (6), one gets:

$$
\begin{aligned}
& \frac{d^{j-f}\left(\left(\lambda^{2}-t \lambda\right)^{n-p-1}\right)}{d\left(\lambda^{2}-t \lambda\right)^{j-f}} \\
& =\left\{\begin{array}{cl}
\frac{(n-p-1) !\left(\lambda^{2}-t \lambda\right)^{n-p-1-j+f}}{(n-p-1-j+f) !}, & \text { if } j-f \leq n-p-1 \\
0, & \text { if } n-p-1<j-f
\end{array}\right.
\end{aligned}
$$

So

$$
\begin{gathered}
\sum_{j=0}^{i-1}(-1)^{j}\left[\widetilde{F}_{p, j} u^{(i-j-1)}(\lambda)\right]_{0}^{t}=0, \\
D_{i, p}(u)=\frac{(-1)^{i}}{(n-p-1) !} \int_{0}^{t}\left\{(t-\lambda)^{n-p-1}(-\lambda)^{n}\right\}^{(i)} u(\lambda) d \lambda
\end{gathered}
$$

Using (9), (13) and (15), one gets the expression of the estimate $y_{e}^{(p)}$ as follows:

$$
y_{e}^{(p)}=\frac{1}{(-t)^{n}}\left(\sum_{i=0}^{m} b_{i} D_{i, p}(u)-\sum_{i=0}^{n} a_{i} \tilde{A}_{i, p}(y)-\Gamma_{p}\left(y_{e}\right)\right)
$$

Thus, one obtains (4). Due to the triangular structure of the matrix $\tilde{R}$, one gets the estimate of the $p-t h$ time derivative of $y$ in function of the integral of $y$ and the input $u$ only.

Step 3: Reconstructor of states

Replacing the relation (3) with the estimated $y_{e}^{(p)}$, one obtains the estimate of the state:

$$
x_{e}(t)=\mathbf{O}_{(A, C)}^{-1}\left[\left(\begin{array}{c}
y_{e} \\
y_{e}^{(1)} \\
y_{e}^{(2)} \\
\vdots \\
y_{e}^{(n-1)}
\end{array}\right)-\mathbf{M}\left(\begin{array}{c}
u \\
\dot{u} \\
u^{(2)} \\
\vdots \\
u^{(n-2)}
\end{array}\right)\right]
$$

Remark 1: Let us mention that all these computations are singular at time $t=0$ but become valid in any arbitrary small instant (see the example in section IV).

The computation of $y_{e}$ is needed for two reasons: firstly, it gives an estimate without noise of the noisy output signal and secondly it is used in the estimation scheme of the successive output derivatives in order to also get rid of the noise effect.

In the above computations, since the successive derivatives of the output depend on integrals (from 0 to $t$ ), the whole signal for these computations is not needed. However, one does not need to keep all output measurements since these integrals can be updated just using the new output values. Thus, these formulas can be adapted to the case of integrals over a sliding window of the signal. 


\section{EXAMPLE: DC MOTOR}

Consider a DC motor system, given by

$$
\begin{cases}\dot{x}_{1} & =x_{2} \\ J \dot{x}_{2} & =K_{1} x_{3} \\ L \dot{x}_{3} & =-R x_{3}-K_{2} x_{2}+u\end{cases}
$$

with $y=x_{1}$ as measured output. $x_{1}$ is the angular position of the rotor, $x_{2}$ is the angular velocity of the rotor, $x_{3}$ is the current of the rotor and $u$ is the control input voltage. $K_{1}, K_{2}, J, L$ and $R$ are strictly positive constant parameters.

\section{A. Algebraic approach}

Write the I/O relationship:

$$
\frac{L J}{K_{1}} y^{(3)}(t)+\frac{R J}{K_{1}} y^{(2)}(t)+K_{2} \dot{y}(t)=u(t)
$$

The state is expressed in terms of the output $y$ as

$$
\left\{\begin{array}{l}
x_{1}=y(t) \\
x_{2}=\dot{y}(t) \\
x_{3}=\frac{J}{K_{1}} y^{(2)}(t)
\end{array}\right.
$$

Step 1: Estimate $y_{e}$ in function of the integral of the output $y$ and the input $u$.

a) Apply the Laplace transform to the relation (16):

$$
\begin{aligned}
& \frac{L J}{K_{1}}\left(s^{3} y(s)-s^{2} y(0)-s \dot{y}(0)-y^{(2)}(0)\right) \\
& +\frac{R J}{K_{1}}\left(s^{2} y(s)-s y(0)-\dot{y}(0)\right) \\
& \quad+K_{2}(s y(s)-y(0))=u(s)
\end{aligned}
$$

b) Derive (17) w.r.t $s$ thrice to eliminate the initial conditions:

$$
\begin{aligned}
\frac{L J}{K_{1}}(6 y(s)+ & \left.18 s \frac{d y(s)}{d s}+9 s^{2} \frac{d^{2} y(s)}{d s^{2}}+s^{3} \frac{d^{3} y(s)}{d s^{3}}\right) \\
+\frac{R J}{K_{1}} & \left(6 \frac{d y(s)}{d s}+6 s \frac{d^{2} y(s)}{d s^{2}}+s^{2} \frac{d^{3} y(s)}{d s^{3}}\right) \\
+ & K_{2}\left(3 \frac{d^{2} y(s)}{d s^{2}}+s \frac{d^{3} y(s)}{d s^{3}}\right)=\frac{d^{3} u(s)}{d s^{3}}
\end{aligned}
$$

Multiply each side of (18) by $s^{-3}$ :

$$
\begin{aligned}
\frac{L J}{K_{1}}\left(\frac{6}{s^{3}} y(s)+\frac{18}{s^{2}} \frac{d y(s)}{d s}+\frac{9}{s} \frac{d^{2} y(s)}{d s^{2}}+\frac{d^{3} y(s)}{d s^{3}}\right) \\
+\frac{R J}{K_{1}}\left(\frac{6}{s^{3}} \frac{d y(s)}{d s}+\frac{6}{s^{2}} \frac{d^{2} y(s)}{d s^{2}}+\frac{1}{s} \frac{d^{3} y(s)}{d s^{3}}\right) \\
+K_{2}\left(\frac{3}{s^{3}} \frac{d^{2} y(s)}{d s^{2}}+\frac{1}{s^{2}} \frac{d^{3} y(s)}{d s^{3}}\right)=\frac{1}{s^{3}} \frac{d^{3} u(s)}{d s^{3}}
\end{aligned}
$$

c) Applying the inverse Laplace transform to (19) and using the formula (ii) to simplify the expression from double integral to simple integral, one obtains the following estimate for $y(t)$.

$$
\begin{array}{r}
y_{e}(t)=\frac{\int_{0}^{t} \frac{(t-\phi)^{2}}{2}\left(\left(6-6 \frac{R}{L} \phi+3 \frac{K_{1} K_{2}}{L J} \phi^{2}\right) y(\phi)+\frac{K_{1}}{L J} \phi^{3} u(\phi)\right) d \phi}{t^{3}} \\
+\frac{\int_{0}^{t}\left((t-\phi)\left(6 \frac{R}{L} \phi^{2}-18 \phi-\frac{K_{1} K_{2}}{L J} \phi^{3}\right)+\left(9 \phi^{2}-\frac{R}{L} \phi^{3}\right)\right) y(\phi) d \phi}{t^{3}}
\end{array}
$$

Step 2: Express $y_{e}^{(1)}, y_{e}^{(2)}$ in function of the integral of $y$. Multiply each side of (18) by $s^{-2}$ :

$$
\begin{aligned}
\frac{L J}{K_{1}}\left(\frac{6}{s^{2}} y(s)\right. & \left.+\frac{18}{s} \frac{d y(s)}{d s}+9 \frac{d^{2} y(s)}{d s^{2}}+s \frac{d^{3} y(s)}{d s^{3}}\right) \\
+ & \frac{R J}{K_{1}}\left(\frac{6}{s^{2}} \frac{d y(s)}{d s}+\frac{6}{s} \frac{d^{2} y(s)}{d s^{2}}+\frac{d^{3} y(s)}{d s^{3}}\right) \\
+ & K_{2}\left(\frac{3}{s^{2}} \frac{d^{2} y(s)}{d s^{2}}+\frac{1}{s} \frac{d^{3} y(s)}{d s^{3}}\right)=\frac{1}{s^{2}} \frac{d^{3} u(s)}{d s^{3}}
\end{aligned}
$$

Multiply each side of (18) by $s^{-1}$ :

$$
\begin{aligned}
\frac{L J}{K_{1}}\left(\frac{6}{s} y(s)\right. & \left.+18 \frac{d y(s)}{d s}+9 s \frac{d^{2} y(s)}{d s^{2}}+s^{2} \frac{d^{3} y(s)}{d s^{3}}\right) \\
+ & \frac{R J}{K_{1}}\left(\frac{6}{s} \frac{d y(s)}{d s}+6 \frac{d^{2} y(s)}{d s^{2}}+s \frac{d^{3} y(s)}{d s^{3}}\right) \\
+ & K_{2}\left(\frac{3}{s} \frac{d^{2} y(s)}{d s^{2}}+\frac{d^{3} y(s)}{d s^{3}}\right)=\frac{1}{s} \frac{d^{3} u(s)}{d s^{3}}
\end{aligned}
$$

b) Return to time domain.

Apply the inverse Laplace transform to (21) and (22), then inject the result of (20) in order to have $y_{e}^{(1)}(t)$ only in function of the integral of $y(t)$ :

$$
\begin{aligned}
& y_{e}^{(1)}(t) \\
& =\frac{1}{t^{3}} \int_{0}^{t}(t-\phi)\left[\left(6-6 \frac{R}{L} \phi+3 \frac{K_{1} K_{2}}{L J} \phi^{2}\right) y(\phi)+\frac{K_{1}}{L J} \phi^{3} u(\phi)\right] d \phi \\
& +\frac{1}{t^{3}} \int_{0}^{t}\left(6 \frac{R}{L} \phi^{2}-18 \phi-\frac{K_{1} K_{2}}{L J} \phi^{3}\right) y(\phi) d \phi+\left(\frac{6}{t}-\frac{R}{L}\right) y_{e}(t)
\end{aligned}
$$

With the result of (20), (23) one gets:

$$
\begin{aligned}
& y_{e}^{(2)}(t) \\
& =\frac{1}{t^{3}} \int_{0}^{t}\left[\left(6-6 \frac{R}{L} \phi+3 \frac{K_{1} K_{2}}{L J} \phi^{2}\right) y(\phi)+\frac{K_{1}}{L J} \phi^{3} u(\phi)\right] d \phi \\
& +\left(\frac{3 R}{L t}-\frac{6}{t^{2}}-\frac{K_{1} K_{2}}{L J}\right) y_{e}(t)+\left(\frac{3}{t}-\frac{R}{L}\right) y_{e}^{(1)}(t)
\end{aligned}
$$

Step 3: Reconstruction of state.

Finally, all the state is expressed in function of $u(t)$ and the integral of $y(t)$ and $u(t)$.

$$
\begin{aligned}
x_{1 e} & =\frac{1}{t^{3}} \int_{0}^{t} \frac{(t-\phi)^{2}}{2}\left(\left(6-6 \frac{R}{L} \phi+3 \frac{K_{1} K_{2}}{L J} \phi^{2}\right) y(\phi)+\frac{K_{1}}{L J} \phi^{3} u(\phi)\right) d \phi \\
& +\frac{1}{t^{3}} \int_{0}^{t}\left((t-\phi)\left(6 \frac{R}{L} \phi^{2}-18 \phi-\frac{K_{1} K_{2}}{L J} \phi^{3}\right)+\left(9 \phi^{2}-\frac{R}{L} \phi^{3}\right)\right) y(\phi) d \phi \\
x_{2 e} & =\frac{1}{t^{3}} \int_{0}^{t}(t-\phi)\left(\left(6-6 \frac{R}{L} \phi+3 \frac{K_{1} K_{2}}{L J} \phi^{2}\right) y(\phi)+\frac{K_{1}}{L J} \phi^{3} u(\phi)\right) d \phi \\
& +\frac{1}{t^{3}} \int_{0}^{t}\left(6 \frac{R}{L} \phi^{2}-18 \phi-\frac{K_{1} K_{2}}{L J} \phi^{3}\right) y(\phi) d \phi+\left(\frac{6}{t}-\frac{R}{L}\right) y_{e}(t) \\
x_{3 e} & =\frac{J}{K_{1}} \frac{1}{t^{3}} \int_{0}^{t}\left(\left(6-6 \frac{R}{L} \phi+3 \frac{K_{1} K_{2}}{L J} \phi^{2}\right) y(\phi)+\frac{K_{1}}{L J} \phi^{3} u(\phi)\right) d \phi \\
& +\frac{J}{K_{1}}\left(\left(\frac{3 R}{L} \frac{1}{t}-\frac{6}{t^{2}}-\frac{K_{1} K_{2}}{L J}\right) y_{e}(t)+\left(\frac{3}{t}-\frac{R}{L}\right) y_{e}^{(1)}(t)\right)
\end{aligned}
$$




\section{B. Simulation}

Hereafter, good estimate and robustness w.r.t noise is depicted. The following parameters are used: $K 1=1(\mathrm{~N} /(\mathrm{m}$. $A)), K 2=1(N /(m \cdot A)), L=0.1(H), R=1(\Omega)$ and $J=$ $5\left(N \cdot s^{2} / m \cdot r a d\right)$ with the initial conditions: $x_{3}(0)=3(A)$, $x_{2}(0)=0(\mathrm{rad} / \mathrm{s})$ and $x_{1}(0)=1(\mathrm{rad})$. The control input voltage is chosen as $u(t)=24$ sint .

Note that all these computations are singular at time $t=0$ but becomes valid for any arbitrary small instant. Therefore one must to evaluate the formula not at time $t=0$ but after a small time $\varepsilon$ (here $\varepsilon=0.05 \mathrm{~s}$ and the state estimation is set to 0 for $t \in[0, \varepsilon])$.
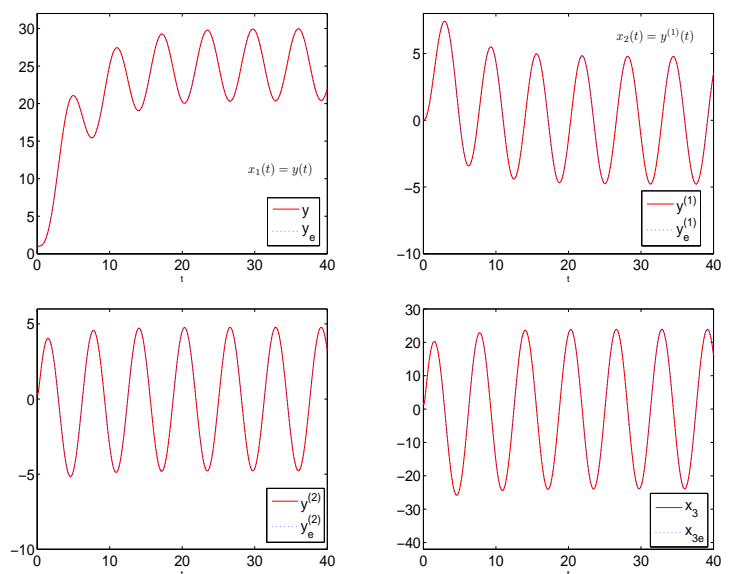

Fig. 1. Original values and its estimates (without noise).
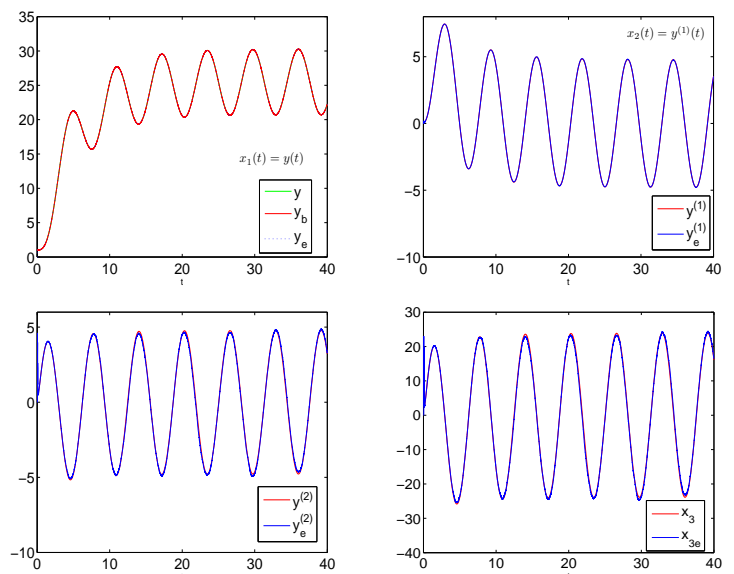

Fig. 2. Original values and its estimates (affected by white noise).

Fig. 1 shows that the estimator performs well because the estimated value tracks exactly the real value after a short time.

In Fig. 2, the measured signal $y(t)$ was perturbed by a white noise (see Fig. 3 where the right figure is a zoom of the noisy output and its estimate) (generated by computer), uniformly distributed in the interval $[-0.015,0.015]$, with a measurement sampling rate of $1000 \mathrm{~Hz}$. It can be seen that this estimator is quite robust w.r.t white noise.
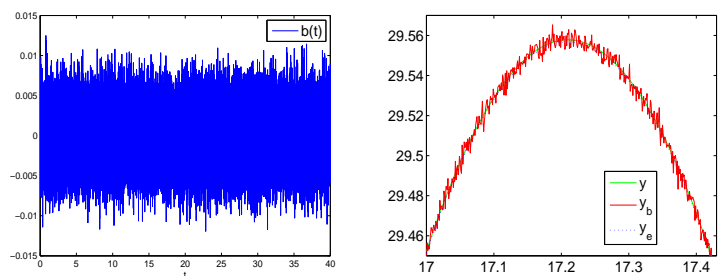

Fig. 3. White noise.

\section{CONCLUSION}

In this paper, an algebraic approach for fast state estimation for linear time-invariant systems has been introduced. Additionally, a generalized exact formula for the estimates has been derived. It should be stressed that this approach exhibits good robustness properties with respect to white noise.

\section{ACKNOWLEDGEMENT}

The authors would like to thanks the reviewers for their valuable comments about this paper.

\section{REFERENCES}

[1] J. P. Barbot, M. Fliess, and T. Floquet. An algebraic framework for the design of nonlinear observers with unknown inputs. 46th IEEE Conference on Decision and Control, 2007.

[2] M. Fliess. Generalized controller canonical forms for linear and nonlinear dynamics. IEEE Trans. Automat. Control, 35:9, 1990.

[3] M. Fliess and S. Diop. Nonlinear observability, identifiability and persistent trajectories. Proc. 36th IEEE Conference on Decision Control, 1991.

[4] M. Fliess and H. Sira-Ramirez. An algebraic framework for linear identification. ESAIM Control Optim., 9:151-168, 2003.

[5] M. Fliess and H. Sira-Ramirez. Closed-loop parametric identification for continuous-time linear systems via new algebraic techniques. In Continuous-Time Model Identification from Sampled Data. Springer, 2008.

[6] R. E. Kalman. A new approach to linear filtering and prediction problems. Transactions of the ASME - Journal of Basic Engineering, 82-D:35-45, 1960.

[7] R. E. Kalman and R. S. Bucy. New results in linear filtering and prediction theory. Transactions of the ASME - Journal of Basic Engineering, 83:95-107, 1961.

[8] D.G. Luenberger. Observers for multivariable systems. IEEE Trans. Automat. Control, 11(2):190-197, 1966. 